\title{
Automatisierungs- und Steuerungssysteme in der Produktion
}

\author{
A. Zoitl, G. Schitter
}

angenommen am 6. August 2021, online publiziert am 24. August 2021

(c) Springer-Verlag GmbH Austria, ein Teil von Springer Nature 2021

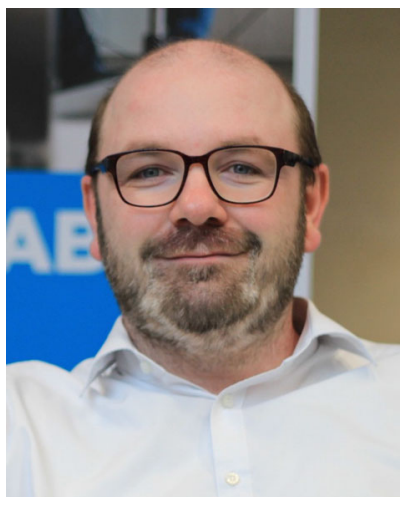

Univ.-Prof. Dipl.-Ing. Dr.techn.

Alois Zoitl

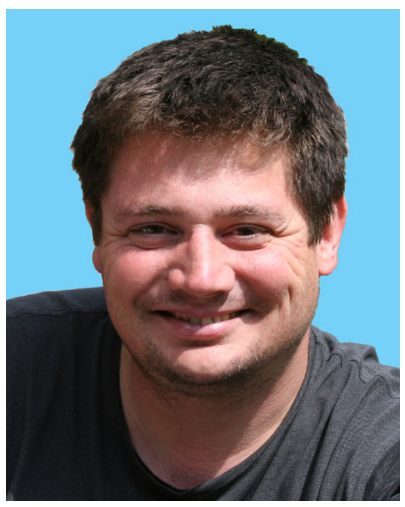

Univ.-Prof. Dipl.-Ing. Dr.sc.techn., Dipl. NDS ETHZ Georg Schitter
Die produzierende Industrie steht vor großen Herausforderungen, da sich die Produktlebenszyklen verkürzen, die Produktvariabilität zunimmt und die globalen Märkte unbeständiger werden. Um schnell und effizient auf diese Veränderungen reagieren zu können und wettbewerbsfähig zu bleiben, müssen Produktionsanlagen und -geräte somit anpassungsfähig sein (Stichwort Industrie 4.0). Eine Schlüsselkomponente zur Erreichung dieser Ziele ist die Automatisierungsund Steuerungsinfrastruktur. Der Umfang der Software in Produktionsautomatisierungssystemen nimmt ständig zu. Verstärkt wird dies durch die Forderung nach einem höheren Vernetzungsgrad dieser Systeme. Derzeitige Technologien stoßen bereits an ihre Grenzen. Dies führt zu steigendem Entwicklungsaufwand und Kosten. Neue Interaktions- und Kommunikationsansätze sowie neue Wege der Program-

mierung von Automatisierungs- und Steuerungssystemen, die aus vernetzten Steuergeräten bestehen, sind notwendig.

Dieses Sonderheft, welches sechs Beiträge umfasst, gibt einen Überblick über neue Ansätze zur Programmierung und Umsetzung von Automatisierungs- und Steuerungssystemen in der Produktion.

Der erste Beitrag dieses Heftes von M. Melik-Merkumians, P. Gsellmann und G. Schitter mit dem Titel Modularisierung von Steuerungssoftware - Effizientes Engineering mit IEC 61131 und IEC 61499 widmet sich dem Thema, wie durch geeignete Entwurfsmuster Steuerungssoftware modularer und wiederverwendbarer umgesetzt werden kann. Der Kern dieses Musters ist ein hierarchischer Ansatz, welcher Steuerungscode von der Hardwaresteuerung entkoppelt.

Im zweiten Beitrag mit dem Titel Software-Qualitätssicherung im Maschinen- und Anlagenbau: automatisierte Bewertung der technischen Qualität von SPS-Code widmen sich B. Dorninger und T. Ziebermayr ebenfalls der Programmierung von Steuerungssoftware. Sie betrachten aber die Möglichkeiten, wie werkzeugunterstützt Steuerungscode analysiert und nach diversen Qualitätskriterien beurteilt werden kann. Dies erlaubt Steuerungsprogrammierer/innen, frühzeitig Rückmeldung zu problematischen Code-Stellen bereitzustellen.

Der Beitrag Ansätze zur Beherrschung der Variabilität in Softwareintensiven Cyber-Physischen Produktionssystemen von A. Gutierrez, L. Sonnleithner, A. Zoitl und R. Rabiser gibt einen detaillierten Überblick darüber, wie die Variabilität von Steuerungssoftware aufgrund der Anforderungen von Produktionsmaschinen und -anlagen abgebildet und durch Ansätze der Variabilitätsmodellierung beherrscht werden kann.

Der vierte Beitrag mit dem Titel Developing an engineering tool for Cyber-Physical Production Systems von U. Kannengiesser, J. Frysak, C. Stary, F. Krenn und H. Müller befasst sich mit der Thematik, wie Cyber-Physische Produktionssysteme von Domänenexpert/innen effizient entwickelt werden können. Insbesondere werden die hierfür notwendige Werkzeugunterstützung entwickelt und die Erfahrungen, welche in einer ersten Nutzung gewonnen wurden, präsentiert.

G. Weichhart, J. Mangler, A. Raschendorfer, C. Mayr-Dorn, C. Huemer, A. Hämmerle und A. Pichler präsentieren in ihrem Beitrag mit dem Titel An adaptive system-of-systems approach for resilient manufacturing, wie resiliente Fertigungssysteme realisiert werden können. Hierbei wird ein Ansatz eines dezentralen, adaptiven Systems verfolgt. Anhand von drei Systemen wird gezeigt, wie mit diesem Ansatz Resilienz gegenüber diversen Veränderungen und Störungen erreicht werden kann.

Im sechsten Beitrag mit dem Titel Distributed tracing of OPC UA method calls präsentieren C. Mayr-Dorn, B. Pereszteghy, J. Holzweber und M. Mayrhofer eine Machbarkeitsstudie, wie die Kommunikation in einem verteilten, auf dem Kommunikationssystem OPC UA basierenden Automatisierungssystem überwacht und aus den gewonnen Daten Rückschlüsse auf den Zustand des Systems gezogen werden können.

Als Gastherausgeber möchten wir uns an dieser Stelle ganz herzlich bei den Autor/innen für die Beiträge zu diesem Sonderheft des GMAR-Fachbereichs Automatisierungs-, Regelungstechnik und Mechatronik sowie bei den Gutachtern für deren exzellenten Einsatz, zeitgerechte und professionelle Gutachten für alle Fachbeiträge zu erstellen, bedanken. Bei dieser Gelegenheit ergeht unser Dank auch an die österreichische Gesellschaft für Mess-, Automatisierungs- und Robotertechnik GMAR (http://www.gmar.at), an den OVE und an das e\&i-Redaktionsteam für die laufende Unterstützung.

Wir als Fachbereich Automatisierungs-, Regelungstechnik und Mechatronik der GMAR hoffen, dass es uns gelungen ist, mit die-

Zoitl, Alois, LIT Cyber-Physical Systems Lab, Johannes Kepler Universität Linz, Altenberger Straße 69, 4040 Linz, Österreich (E-Mail: alois.zoit|@jku.at); Schitter, Georg, Institut für Automatisierungs- und Regelungstechnik, Technische Universität Wien, Gußhausstraße 25-29/E376, 1040 Wien, Österreich 
sem Heft einen Einblick in den aktuellen Stand der Technik von Softwaresystemen für Automatisierungs- und Steuerungssysteme in der Produktion zu geben und die Wichtigkeit dieses Themas zu vermitteln.
Hinweis des Verlags Der Verlag bleibt in Hinblick auf geografische Zuordnungen und Gebietsbezeichnungen in veröffentlichten Karten und Institutsadressen neutral. 